\title{
The Looking Glass: Visually Projecting Yourself to the Past
}

\author{
Bon Adriel Aseniero and Ehud Sharlin \\ Interactions laboratory, uTouch group, \\ Department of Computer Science, University of Calgary \\ 2500 University Drive NW, Calgary, Alberta, Canada, T2N 1N4 \\ \{baasenied, ehudacpsc\}ucalgary.ca
}

\begin{abstract}
Memories define us as individuals and are considered a special aspect of one's life. We try to preserve our memories as much as possible by taking pictures, videos, or through any form of documentation that we can revisit later on in our lives. Our goal is to design an immersive system that allows rich documentation of the past and interactive 3D-revisitation of the stored reflections in the future. Our prototype system, the Looking Glass captures human experiences and allows users to interactively explore them. Our system, unlike classic methods of documentation, extends the experience of visiting memories beyond viewing, and affords an immersive interactive experience. The Looking Glass is envisioned to provide a 3D visual experience of revisiting past scenes, allowing a more entertaining and emotionally engaging personal approach to re-experiencing past memories. This short paper presents our research motivation and design approach, details our implementation efforts, and current prototype.
\end{abstract}

Keywords: affective interaction, immersive media, interactive art, 3D scene extraction, 3D motion capture, time travel, self-reflecting entertainment computing.

\section{Introduction}

Documenting the past has been a part of human society since the dawn of civilization. Humans were, and still are, documenting their lives with any media accessible to them, from cave drawings to books, journals, and so forth. Our current technology provides plentiful means of documenting the past, such as pictures and videos. We document our past so that we can revisit our past experiences at a later time, to be able to recollect them in case we forget, or to be able to contribute and share them with our various communities, or in order to contribute to a collective knowledge. However, the most common reason for documenting is often very personal; we want to be able to hold on to moments for a little longer, to be able to somehow revisit a cherished memory.

With our current technology, our methods of revisiting the past are limited to viewing, listening, or reading, and the rest is left to one's imagination. A person may be content to just view the past, but we argue that the ultimate way of revisiting it is through time travelling (regardless of whether this is a physically impossible 


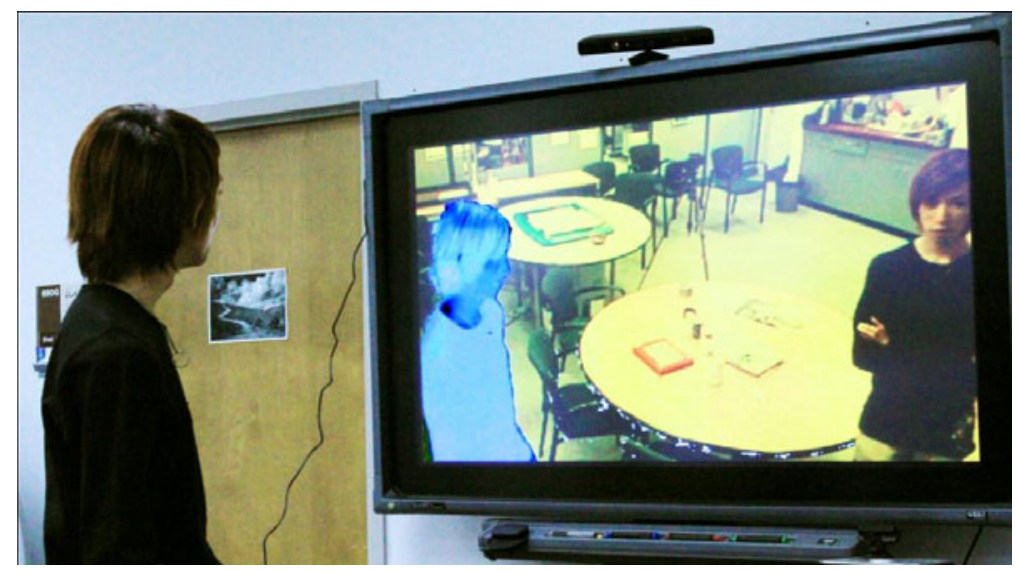

Fig. 1. The Looking Glass is an interactive installation exploring a limited time traveling technique. It projects a user to a captured scene in real-time.

endeavor). Through time travel, a person will be able to see and re-experience a memory first hand. One will be able to encounter past entities, travel to places which may have been physically altered over the years and visit them in their past form, and meet and interact with people that occupied these past spaces and may have been long gone since. We believe that being able to interact and participate while reflecting on past memories will be dramatically more engaging than simply viewing a past scene, or hearing or reading about it. While our current exploration goal is to seek the artistic and entertainment value of this idea, it may also lead to psychological and rehabilitative applications, such as assisting people dealing with painful past events in their lives and with posttraumatic stress disorder (PTSD) [6], or perhaps, maybe helping people with conditions affecting memory such as Alzheimer's disease. We believe that through active presence and immersion, users will have a higher emotional engagement when they revisit past memories, making the experience more engaging and personal.

However, we currently do not have the physical means to travel back and forth in time, thus revisiting the past in the said manner is practically impossible. As a substitute, we are designing the Looking Glass, an interactive installation which attempts to explore a limited variant of time travelling.

Inspired by scenedows and slow glass, in Bob Shaw's Light of Other Days [7], which are engineered glasses used to trap light for a definite amount of time allowing it to capture reflections of sceneries and or people, thus creating an illusion of a 'captured scene', we are designing an interface that attempts to perform this 'capture a scene' action. Since our inspiration emerged from the story's physical form of glass, we designed our system such that it allows its users to see their reflection in the scene itself. Our prototype is named the Looking Glass in lieu of Lewis Carroll's novel, Through the Looking-Glass, where in the main character, Alice, enters an entirely different world, which is somewhat reflective of her own, through a mirror [2].

In this paper we present our current prototype of the Looking Glass, an interactive $3 \mathrm{D}$ documentation and revisitation installation which is designed to provide a limited time travel-like experience, enabling 3D interactive revisitation of past memories 
(Fig. 1). We believe and argue that the ability to project oneself into the past in a rich, 3D immersive manner can open up novel layers of revisiting memory, well beyond simple viewing.

\section{Related Works}

We detail here a few related works that address the issue of Time and its representations. Jussi Ängeslevä and Ross Cooper created a system called Last Clock [1] which displays a space's history and rhythm through an analog clock-like visualization of time. According to them, the created mandalas of archived time show an overview of a given space's dynamics, history, and complex information such as captured activities and motion. These are continuously updated and can be used to inform people who are unfamiliar with certain spaces about a space's dynamics.

Another relevant effort is Gutwin's Traces [3], - a visualization of past movements which helps users gain interactive awareness and perceive one another more clearly and accurately. Gutwin's work was later extended in Timeline [5], a visualization that allows users to rapidly explore the history of a video stream to gain better insight about the activities and availability of other people.

The Family Window [4] is a domestic media space that allows the connections of separated families through video connection. The Family Window has a 'time shift' capability which records videos containing activities and then sends it to the other household for viewing at a later time. Through the 'time shift' mode, families separated by time zones can pretend to be at the same time and place. Similarly, $C U$ Later [8] was designed to by-pass the time difference between two locations to connect people together.

While these projects are closely related to our work, we believe that the Looking Glass is unique. The Looking Glass is an attempt to visualize the past in an interactive fashion not for communication purposes, but rather for self-reflection. The visitor's experience is the focal point of our design. The past entities being revisited, places, and people, may be long gone, and we are not trying to by-pass time to communicate with them. The Looking Glass also attempts to push the revisitation experience into the realm of 3D immersion, employing 3D scene mapping and motion capturing techniques to create a revisitation experience which is as interactive as possible.

\section{Design and Implementation}

Our current prototype for the Looking Glass is designed to allow real-time capturing and storing of past scenes, as well as a later projection of a visitor into the past scene through augmented reality. Our current prototype is using the Microsoft Kinect, with its available OpenNI (Open Interaction) development kit ${ }^{1}$, and is coded in C\# WPF. The Looking Glass prototype makes use of the Kinect's ability to capture video feed and depth information. The video feed combined with the range data allows future reconstruction and $3 \mathrm{D}$ rendering of the previously captured scenes.

\footnotetext{
${ }^{1}$ From the OpenNI website: http://www.openni.org
} 
For the Looking Glass' to be an integral component of people's lives, we envision that it should be able to capture scenes "infinitely" such that a person can browse and ultimately revisit any point in the past, within the physical confines of the system's field of view. Once installed, the Kinect should no longer be moved, and is capturing the scene continuously. Also, the system should be non-intrusive; almost invisible to the people being captured, to remove the awkward feeling of being watched or recorded which may affect the genuineness of a scene being captured. With this in mind, we have implemented the Looking Glass to act like a mirror with a computer monitor acting as the mirror itself, while the Kinect sits nearby acting as a sort of physical ornament (see Fig. 1). Through this setup the Looking Glass acts as ubiquitous furniture, a part of the room itself; this makes it non-intrusive.

The main functionalities of the Looking Glass' current prototype are capturing and revisiting. Our current design does not include audio capturing, a feature that is supported by the Kinect, and we are hoping to integrate in our future design.

Capturing. The Looking Glass, in its current prototype is able to capture scenes and its corresponding depth information. However, this is far from the envisioned system which captures scenes infinitely. We currently do not have any means of recording data infinitely; hence, we have designed the Looking Glass such that it only captures scenes when instructed to do so. Although this limits users to only be able to revisit select scenes from the past - those which were captured, we believe that it still allows us to explore our design concept and gain user feedback. Once a satisfying amount of data has been captured, the user can choose to stop capturing. After capturing, the system will create an ONI type file (OpenNI's file format that can contain a Kinect video feed and corresponding depth information) labeled with the time it was created. These files are used by the Looking Glass for later revisits to past scenes.

Revisiting. The current prototype of the Looking Glass we present here is able to let users view previously captured scenes, and is able to project them as visitors into the scene which is currently being viewed. It maps the visiting user, with regards to depth information, into the past scene being visited (Fig. 1 and 2). However, it still does not support 3D rendering, limiting the exploration to the static point of view of the Kinect. In a short term, we are planning to implement 3D rendering of scenes to enable the visitor to explore the scene from any point of view as they wish to.

Although the current prototype does not render in $3 \mathrm{D}$, the recorded scene is accompanied by corresponding depth information from the Kinect allowing the prototype to project a visitor into the scene in accordance with their spatial relationship to that of the past scene. It allows the prototype to occlude the users whenever there is an entity in the captured scene that should be occluding them based on their location. In essence, while in viewing mode, the visitor is able to walk around and experience the scene while their current-time projected image on the scene may be dynamically occluded by past scene entities. This arguably allows the user to visually blend with the past scene, creating an illusion that they are a part of it.

We have also incorporated a function within the view mode which colorizes the past feed and the present feed (see Fig. 2). This separates the present and the past in a more visually distinctive manner (Fig. 1 and 2). 


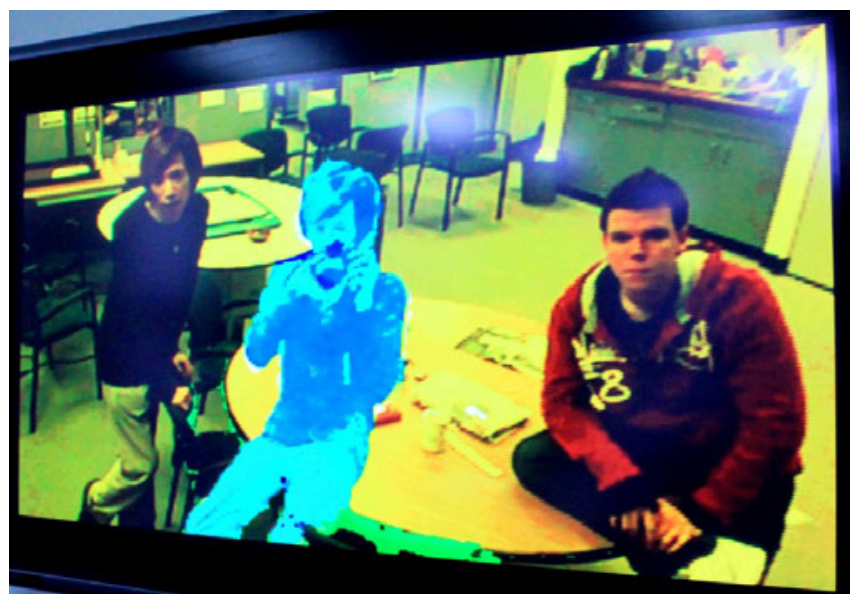

Fig. 2. The Looking Glass projects a user into the captured scene as a ghost-like blue figure, while the captured scene is tinted yellow for an 'old sepia photo' effect

\section{Preliminary Evaluation}

No formal studies have been conducted yet to evaluate the current prototype for the Looking Glass. The following evaluation of the prototype is based on a preliminary design critique of the system done with a few members of our lab. The current prototype of the Looking Glass is still far from our time travel vision. Interacting with it in its current phase is an interesting visual experience, but quite limited, arguably not that different from viewing a video.

However, we did observe that the interesting spatial interaction with past entities makes the interaction with the Looking Glass interesting and entertaining even in its current limited form. Visitors can be obscured dynamically and occluded by past entities or even past replicas of themselves. Alternatively, visitors can also obscure and occlude past entities, or emerge by spatially walking through them (i.e. visitors visually entering the scenes by penetrating through their past selves.) Moreover, because any physical activities can be captured and revisited, we see applications to our approach for documenting and following personal progress, for example when learning a new physical activity such as a baby's first steps, a set of new dance moves, or a sequence of Karate Kata movements.

\section{Future-Work and Conclusion}

Humanity's interaction with time, as well as time travelling has always been and will still be a topic of philosophical exploration, and science fiction. While we cannot physically travel back in time, emergent technologies and techniques are now available such that we can develop a system that allows us to mimic time travelling. In this paper we have presented our attempt to pursue this goal, expressed through the Looking Glass' design approach and current prototype. 
Over all, the current prototype of the Looking Glass is lacking in necessary functionalities that would bring it close to mimic time travelling. However, we believe that the current prototype hints at the potential of our approach, and we hope that within the next iteration of this prototype, we will be able to demonstrate a $3 \mathrm{D}$ immersive approach that will change the way users revisit memories, making the experience more personal and engaging.

Taking note of the problems found through the simple design critique of our prototype, we are currently working on the next prototype of the Looking Glass. The most important feature that we will be integrating into the system is the $3 \mathrm{D}$ reconstruction of scenes, giving the visitors the ability to change point of views. We believe that this will allow a dynamic, physical exploration of the past scene, making revisiting the past a more genuine and engaging experience. Another change we are exploring is to support a more aesthetically pleasing color palette when viewing on colorized form; for example, colors that have been selected with regards to art color theory. Also, we are exploring ways of incorporating sound into captured scenes.

Through these, re-experiencing the past can become a more informative and engaging, and closer to the goal of mimicking time travel.

\section{References}

1. Ängeslevä, J., Cooper, R.: Last Clock. IEEE Computer Graphics and Applications 25, 20$23(2005)$

2. Carroll, L.: Through the Looking Glass. Macmillan, United Kingdom (1871)

3. Gutwin, C.: Traces: Visualizing the Immediate Past to Support Group Interaction. In: Proc. Graphics Interface, pp. 43-50 (2002)

4. Judge, T.K., Neustaedter, C., Kurtz, A.F.: The Family Window: The Design and Evaluation of a Domestic Media Space. In: Proceedings of the 28th International Conference on Human Factors in Computing Systems, Atlanta (2010)

5. Nunes, M., Greenberg, S., Carpendale, S., Gutwin, C.: Timeline: Video Traces for Awareness. In: CSCW 2006, Banff (2006)

6. Rizzo, A., Reger, G., Gahm, G., Difede, J., Rothbaum, B.O.: Virtual Reality Exposure Therapy for Combat-Related PTSD. Post-Traumatic Stress Disorder. Part 6, 375-399 (2009)

7. Shaw, B.: Light of Other Days. Analog (1966)

8. Tsujita, H., Yarosh, S., Abowd, G.D.: CU-Later: A Communication System Considering Time Difference. In: UbiComp 2010, Copenhagen (2010) 